\title{
Short-term proactive interference revisited
}

\author{
A. J. P. HENDRIKX \\ Tilburg University, Tilburg, The Netherlands
}

(A. F. Sanders, Sponsor)

\begin{abstract}
The basic experimental task consisted of memorization of a list of consonants followed by positionally probed recall of a single item. Immediately after presentation of a list, a positional probe was presented indicating the serial position (SP) of the requested item. Response latency was the main dependent measure. Previous studies with this paradigm have shown that if many trials are run in close succession, the latency for the most recent SP is smaller than that for the first SP, whereas the reverse is found when trials are given in relative isolation. The hypothesis is tested that this effect reflects a buildup of proactive interference (PI) over successive trials. Subjects received strings of six closely spaced trials, and the effect of successive trials on RT was measured. In line with the common findings on PI in short-term recall, the main effect of PI occurred at the second trial of a string, without any clear effect at later trials. Hence, the relatively strong recency effect as observed with many closely spaced trials seems not to be due to PI but to a more aspecific change in resource allocation to the various parts of the list.
\end{abstract}

In short-term serial recall, performance rapidly declines across trials, due to proactive interference (PI) from preceding trials (e.g., Keppel \& Underwood, 1962). PI builds up as a negatively accelerated function across trials, the bulk of PI stemming from the one or two immediately preceding trials. Furthermore, the buildup appears to depend on temporal proximity: there is no PI when two trials are separated by an interval longer than 2 min (Loess \& Waugh, 1967).

Most studies on short-term PI have employed the socalled Brown-Peterson paradigm, in which a consonant trigram is recalled after a filled retention interval. Yet PI is also observed in the response latency of positionally probed vocal recall of a single item (Sanders \& Willemsen, 1978). In this paradigm, a list of items is followed by a positional probe, indicating the serial position (SP) of the requested item. Usually, response latency is the dependent measure. This paradigm avoids effects of output interference, and moreover, latency may provide a more sensitive measure of PI than does probability of correct recall.

Sanders and Willemsen (1978) observed that in positionally probed recall, only the first items in the list were vulnerable to PI. In their study, two trials were presented in close succession and trial pairs were separated by a longer interval during which PI could dissipate. Thus, the task consisted of alternating non-PI and PI trials (Turvey \& Weeks, 1975). Reaction times (RT) for the first few items of the list increased in the second trial as compared with reaction times in the first trial. The results of Sanders and Willemsen's study comply with PI effects in free

This study was supported by The Netherlands Organization for the Advancement of Pure Research, Z.W.O., Grant 152611. Requests for reprints should be sent to Paul Hendrikx, Department of Psychology, P. O. Box 90153, 5000 LE Tilburg, The Netherlands. recall (Craik \& Birtwistle, 1971). Sanders and Willemsen also found that in the first trial of a pair, RT to the final item of a list was longer than RT to the first item, whereas in the second trial RTs to these positions had about equal latencies. This change from a primacy advantage in non-PI trials to about equal latencies in PI trials was replicated by Hendrikx (1983), again in a setting in which two trials were run in close succession, followed by a longer interval before presenting the next pair. However, in the case of many successive trials, Hendrikx (1984a, 1984b) repeatedly found a substantial faster RT for the most recent item as compared with RT for the first item, the difference amounting to about $200 \mathrm{msec}$. This could suggest that the effect of PI on recall latency accumulates over a larger number of trials than indicated by the traditional measures of recall accuracy.

Therefore, in this paper, we investigate the buildup of PI within strings of six closely spaced trials. Latency of vocal recall was measured in response to a positional probe. Successive strings were separated by longer intervals, allowing for dissipation of PI. The primary question is whether PI continues to build up across trials within a string. PI effects are assessed as a function of trial number, that is, the position of the trial within a six-trial string. The first trial of each string is regarded as free from PI, whereas the second and later trials may increasingly be affected by PI originating from preceding trials (see Turvey \& Weeks, 1975). As a result, faster $R T$ to the first item may gradually change, across trials, into faster RT to the final item.

In addition, effects of list length (LL) and previous list lengths (PLL) were examined. Following the "acid bath" theory of PI (Posner \& Konick, 1966), vulnerability of the recall of the first item to interference from a previous list may increase as the present list grows longer, because the retention interval grows longer. In the same vein, a 
larger length of the preceding list(s) adds to the "concentration of the acid," resulting in a stronger PI effect. The acid bath theory considers PI as an effect on storage. Alternatively, PI may be due to confusion at retrieval. For example, positional cuing theory (Sanders, 1975) explains PI in terms of confusion between cues of successive lists which provide access to the first item. Hence, positional cuing theory does not predict more PI with a longer LL or PLL. Indeed, Sanders and Willemsen (1978) did not observe an effect of either PLL or LL in the case of only one prior interfering list. The present use of sixtrial strings could provide a more powerful test between the notions of acid bath and positional cuing theory, in particular if PI would accumulate across six trials.

\section{METHOD}

\section{Subjects and Task}

Two groups of 4 students of Tilburg University served as subjects. The subjects had no previous experience in memory experiments and were paid a fixed amount and an additional bonus for each correct response. The task was immediate vocal recall of a single item from a list of either 4,5 , or 6 successively presented visual items. The serial position (SP) of the requested item was indicated by a positional probe, consisting of the onset of a light from a horizontal row of lights. Subjects were asked to respond to the probe as quickly and accurately as possible. Subjects vocalized the list in synchrony with presentation. They were asked to vocalize the items at presentation, without stressing or changing voice inflection. This was monitored by the experimenter.

In a trial, a list of consonant letters was visually presented at a rate of 2 letters per second, followed by a 500 -msec interval and a subsequent probe signal. The probe was a light at one of the locations in a horizontal row of 4,5 , or 6 dots, the number of dots corresponding to the number of items in the current list. The dots respectively indicated, from left to right, the first through last item of the list, so that the location in which the probe occurred indicated the SP of the requested item. Successive trials within a string were separated by a 3-sec interval. Subjects performed individually in strings of six closely spaced trials. To allow for dissipation of PI, a pause of $4.5 \mathrm{~min}$ intervened between successive strings; subjects spent the time in leisure outside the experimental room.

A subject was seated in a dimly lit sound-attenuating room, viewing a scope display (DEC GT-40) at a distance of about $1.3 \mathrm{~m}$. A trial started with a 500 -msec auditory warning signal $(2900 \mathrm{~Hz}, 65 \mathrm{~dB})$ and a $1-\mathrm{sec}$ interval, after which a list of either 4,5 , or 6 successive capital letters was presented in the center of the screen, marked by a fixation point. The actual number of items at a particular trial was indicated below the center by the horizontal row of 4,5 , or 6 dots. The dots were presented together with the warning signal and remained on display during the trial. After presentation of the last item, there was an interval of $500 \mathrm{msec}$, followed by the positional probe. The probe consisted of a cursor (a luminous square of $3 \times 2 \mathrm{~mm}$ ) centered at one of the dots in the horizontal row. For each LL, all SPs were probed with equal frequency.

\section{Design and Procedure}

The variables list length (LL), serial position of the probe (SP), trial number $(\mathrm{N})$, and length of the immediately preceding list (PLL) were varied orthogonally within subjects in a $3 \times \mathrm{SP} \times 6 \times 3$ design (SP range depending on LL).

Four subjects at a time participated in three consecutive daily sessions. Each session consisted of 50 6-min periods, during each of which all 4 subjects performed individually and in a fixed order, in a string of six trials. Thus, each subject participated over 3 days in a sequence of 150 strings of six trials each. Each subject received a different order of the same 150 strings. In each session, a 30-min pause interrupted the procedure after the 12 th, 24 th, and 36 th strings. LL was pseudorandomly assigned to trials within strings.
After completion of a trial pair, a subject was informed about the correctness of recall and whether RT fell within acceptable limits (90-2700 $\mathrm{msec})$. The day before the first session, subjects practiced immediate serial written recall of six-letter lists, spoken at a rate of 2 letters per second. The same consonants were used as in the experimental task. Subjects also had 30 trials of practice on the actual experimental task.

\section{Materials}

All lists consisted of a pseudorandom sequence of capital letters, drawn without replacement from the consonant set F, J, K, L, N, P, R, T, $Z$. The frequency of the consonants was counterbalanced over SPs, and all consonants were probed with about equal frequency. This was done separately for each combination of list length and trial number. For the second group of 4 subjects, the letter composition of the lists was changed systematically by a one-to-one replacement of each letter within the set.

Lists of 4,5 , and 6 letters occurred respectively in 40,50 , or 60 trials at each trial number. Thus, for each trial number, the proportion of trials with a given list length was fixed, respectively, at $0.27,0.33$, and 0.40 . In addition, $L L$ was assigned to trials so that the conditional probability of receiving a certain list length at a given trial, given any previous list length, was always equal to the previously mentioned overall proportions.

\section{RESULTS AND DISCUSSION}

The ANOVAs reported below were performed on individual median RTs of correct recall. Effects of PLL were absent, as shown in ANOVAs on the first three SPs, run separately for each of Trials 2 to 6 . Factors were $\mathrm{LL}$ $(4,5,6)$, PLL $(4,5,6)$, and SP $(1,2,3)$. In each ANOVA, there were only significant main effects of LL [ $F$ values for trial numbers 2 through 6 were, respectively, 4.3, 2.4 (nonsignificant), 3.8, 11.7, and 8.3; $d f=2,14$; $p \leq .05$ ] and of SP $(F$ values for trial numbers 2 through 6 were, respectively, 58.9, 49.4, 64.2, 36.8, and 53.8; $d f=2,14 ; p<.001)$. The PLL effect never reached significance, either as main effect or in interactions $[F(2,14)$ $=0.21,0.14,1.2,1.3$, and 1.3 for trial numbers 2 through 6 , respectively]. Hence, data were collapsed across PLL levels. In all conditions, recall errors were quite infrequent and not analyzed.

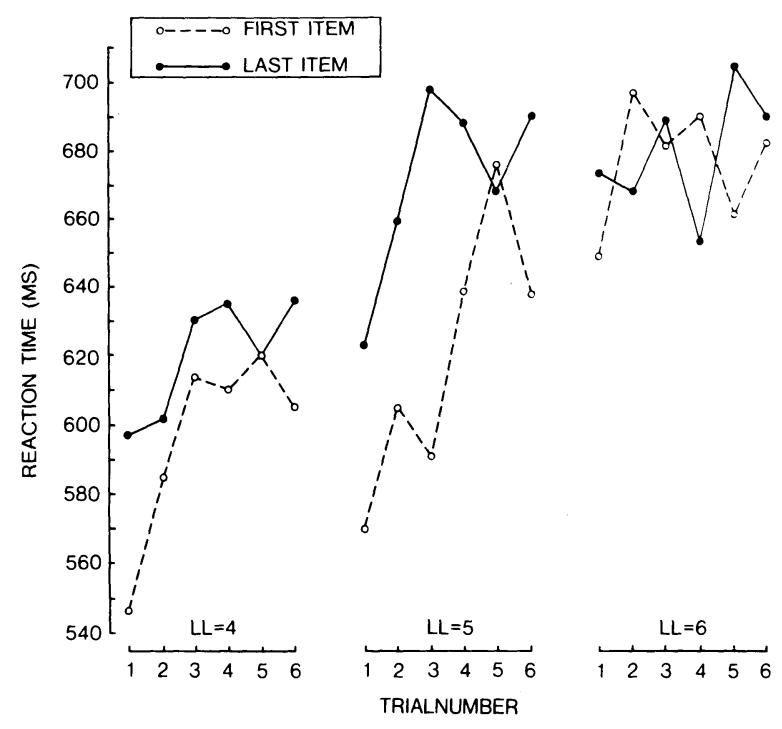

Figure 1. Means of individual median RTs of the first and last serial position (SP) as a function of trial number and list length $(L L)$. First SP: open circles, dashed lines; Last SP: filled circles, solid lines. 


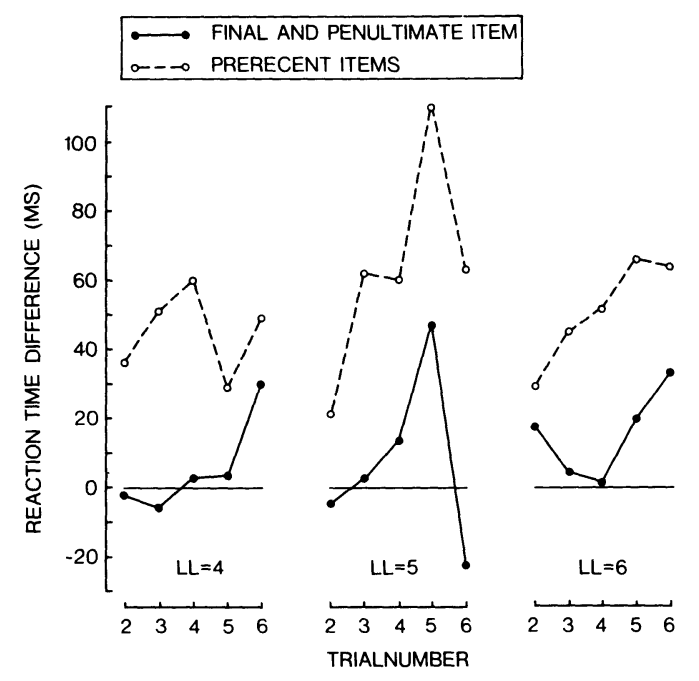

Figure 2. Difference in median RTs between a later trial and the first trial, as a function of trial number and list length (LL), for the pooled early SPs (open circles, dashed lines) and the last two SPs (filled circles, solid lines).

All SP curves for median RTs showed the familiar inverted-U shape. Figure 1 shows means of individual median RTs for the first and last SPs as a function of trial number. In particular, for lists of 4 or 5 items, there was at the early trials a smaller latency for the first item, as compared with latency for the final item, an effect which disappeared at later trials. An advantage for the final item was not observed in any part of the trial string. An ANOVA was run separately for each list length on the first and last SP, with SP and trials (1-6) as factors. The main effect of SP was not significant, but there was a significant effect of trials for LL 4 and LL $5[F(2,35)=5.1$, $p=.002 ; F(2,35)=6.2, p<.001$, respectively]. For LL 5, the SP $\times$ trials interaction was also significant $[F(5,35)=2.6, p=.04]$. Although there was a similar trend at $L L 4$, there was no significant interaction for LL 4 or for $\operatorname{LL} 6[F(5,35)=0.8$ and 1.9 , respectively $]$.

Figure 2 shows the differences in median RT between each of the later trials and the first trial of the six-trial strings, calculated separately for the pooled prerecent items and the last two items. The differences can be used to assess PI. The latter type of difference should reflect trial effects unrelated to PI. PI is reflected in the generally greater difference score for prerecent items observed across all trials, suggesting that the major effect of PI occurs at the second trial. In addition, there is a general trend toward an increase of the differences across trials, which suggests that not all trial effects are related to PI. An ANOVA was run, separately for each list length, on individual differences between later trials and Trial 1 , with trials (2-6) and list part (prerecent vs. last two items) as factors. For LL 5, the effect of trials was significant
$[F(4,28)=5.9, p=.002]$, whereas the effect of list part was only marginally significant $[F(1,7)=3.8, p=.08]$. However, for LL 6 and LL 4, there was no significance for the main effects and the interaction. Since PI effects were rather small, the present data do not allow a rigid test of whether (previous) list length affects the size of PI.

In summary, we obtained no evidence for a continuing PI buildup in the primacy part of the list that could account for the faster RT for the most recent items as compared to the first item, as observed at long series of trials. Also, previous list length does not affect PI, which confirms the results of Sanders and Willemsen (1978). It appears that a global task feature, such as massed versus spaced trials, causes a substantial change in relative performance between list parts which is not due to PI but to a shift in attentional bias favoring, respectively, the last or the first part of a list. In agreement with Hockey, MacLean, and Hamilton (1981), we propose that prolonged task execution may be a stressor that changes the balance of resources for underlying component processes. For example, with massed trials, acquisition of new input may improve at the cost of reduced storage, causing a trade-off in performance between the first and last part of a memory list.

\section{REFERENCES}

Craik, F. I., \& BiRTwistle, J. (1971). Proactive inhibition in free recall. Journal of Experimental Psychology, 91, 120-123.

HENDRIKX, A. J. P. (1983). On the origin of proactive interference in short-term retention. Unpublished manuscript, Tilburg University.

HENDRIKX, A. J. P. (1984a). Effects of grouping on recall latency. Acta Psychologica, 55, 1-18.

HeNDRIKX, A. J. P. (1984b). Temporal aspects of retrieval in shortterm retention. Acta Psychologica, 57, 193-214.

Hockey, R., Maclean, A., \& Hamilton, P. (1981). State changes and the temporal patterning of component resources. In J. Long \& A. Baddeley (Eds.), Attention and performance IX (pp. 607-621). Hillsdale, NJ: Erlbaum.

KePPEL, G., \& UNDERWOOD, B. J. (1962). Proactive inhibition in shortterm retention of single items. Journal of Verbal Learning \& Verbal Behavior, 1, 153-161.

LoESs, H., \& WAUGH, N. C. (1967). Short-term memory and intertrial interval. Journal of Verbal Learning \& Verbal Behavior, 3, 362-368.

PoSNER, M. I., \& KonICK, A. F. (1966). On the role of interference in short-term memory. Journal of Experimental Psychology, 72, 221-231.

SANDERS, A. F. (1975). Some remarks on short-term memory. In P. M. A. Rabbitt \& S. Dornic (Eds.), Attention and performance $V$. New York: Academic Press.

SANDERS, A. F., \& Willemsen, E. M. (1978). The course of proactive interference in immediate probed recall. Acta Psychologica, 42, 133-144.

TURVEY, M. T., \& WEEKS, R. A. (1975). Effects of proactive interference in immediate serial recall. Quarterly Journal of Experimental Psychology, 27, 47-62.

(Manuscript received for publication April 28, 1986.) 